\title{
Machine Learning Based Precision Orientation and Strain Mapping from 4D Diffraction Datasets
}

\author{
Renliang Yuan ${ }^{1}$, Jiong Zhang ${ }^{2}$, Lingfeng $\mathrm{He}^{3}$ and Jian-Min Zuo ${ }^{4}$ \\ ${ }^{1}$ University of Illinois at Urbana-Champaign, Illinois, United States, ${ }^{2}$ Intel Corp, United States, \\ ${ }^{3}$ Idaho National Laboratory, Idaho Falls, Idaho, United States, ${ }^{4}$ University of Illinois at Urbana- \\ Champaign, Urbana, Illinois, United States
}

Four-dimensional diffraction datasets (4D-DDs) collected using the scanning electron nanodiffraction (SEND) [1] or 4D scanning transmission electron microscopy (4D-STEM) [2] techniques have gained increasing popularity in the electron microscopy community for their versatility in both electron diffraction and imaging. The advantage of having a 4D-DD over the traditional 2D imaging techniques comes from the rich information captured in diffraction patterns, which can be related to the sample structure, electric and magnetic fields. Experimental acquisition of large 4D-DDs has been significantly improved with the development of fast electron pixel array detectors [3]. Now, the large data size also brings new challenges to the 4D-DD analysis. Faster and better ways to extract information from 4D-DDs are both needed.

Machine learning (ML) has revolutionized scientific research, which once perceived as a niche subject of computer science has been transformed as a potent technology as demonstrated by the success of AlphaGo [4]. In computer vision, deep learning is used to solve difficult problems, such as detection, classification, and segmentation [5]. In the field of electron microscopy, deep learning has been applied to, or proposed for, crystallographic analysis of electron image and diffraction data [6], single atom detection [7], automatic learning of microstructural features [8], etc.

Here, we describe the design, testing, and benchmarking of two types of ML based analysis of 4DDDs. The first is precision crystal orientation mapping using a trained artificial neural network $(\mathrm{ANN})$, where we determine small changes in crystal orientation within a crystalline grain (Figure 1). We design a simple ANN model to be trained on the simulated dynamical diffraction patterns for electron nanodiffraction with non-overlapping diffraction disks. Using the trained ANN to recognize the intensity distribution among diffraction disks, a high angular resolution of $0.01^{\circ}$ or better is achieved for orientation mapping, which we demonstrate on both single crystalline and polycrystalline samples (GaSb thin sample with the bending contour contrast and irradiated $\mathrm{UO}_{2}$ ). Figure 1 shows multiple small grains sharing low-angle grain boundaries less than $2^{\circ}$ in $\mathrm{UO}_{2}$ with the grains clearly resolved.

The second type of ML based analysis uses a convolutional neural network (CNN) to measure diffraction disk positions in diffraction patterns, which are then used to calculate lattice strain maps (Figure 2). A diffraction pattern is first divided into sub-images containing individual diffraction disks, and the trained $\mathrm{CNN}$ is then used to detect the peak position. The use of sub-images simplifies the CNN structure and expedites both model training and processing of 4D-DDs. The precision and computing efficiency are demonstrated in the measurement of the strain fields in a Si-based FinFET device. The results are comparable with those calculated by the circular Hough transform method [9] and have better precision $(\sim 0.04 \%)$ in some cases. The total processing speed, however, is improved by a factor of 4 or more using the CNN method. 
The above results show that by training neural networks with accurate electron diffraction simulation using dynamical diffraction theory, supervised ML based analysis of large 4D-DDs can provide rich information about nanoscale crystalline materials with both high spatial resolution and high precision.
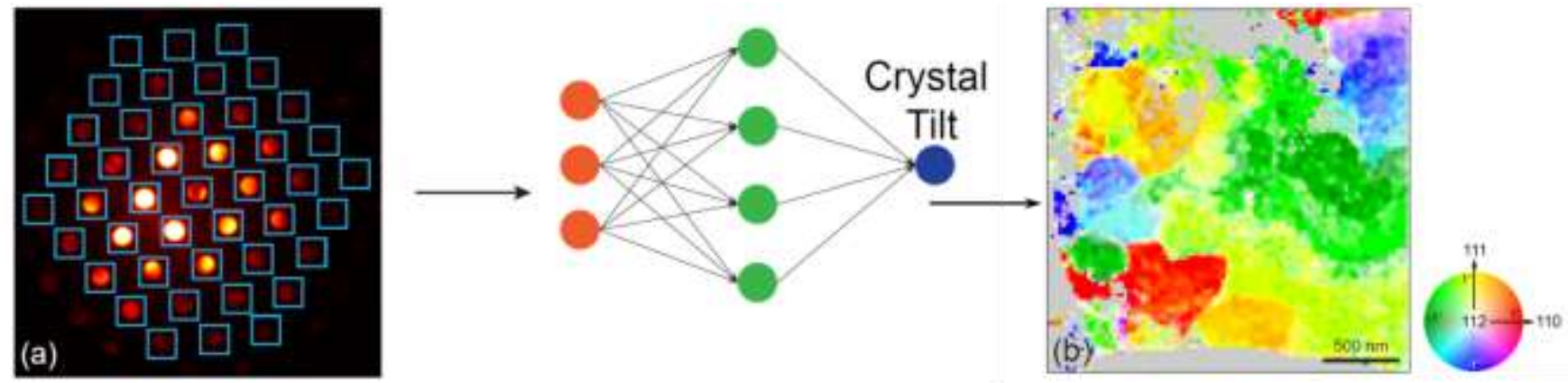

Figure 1. Training artificial neural networks for precision crystal orientation mapping. (a) The design of an ANN for precision crystal orientation determination based on integrated diffraction intensities. (b) Precision orientation mapping of grain subdivision in irradiated UO2.

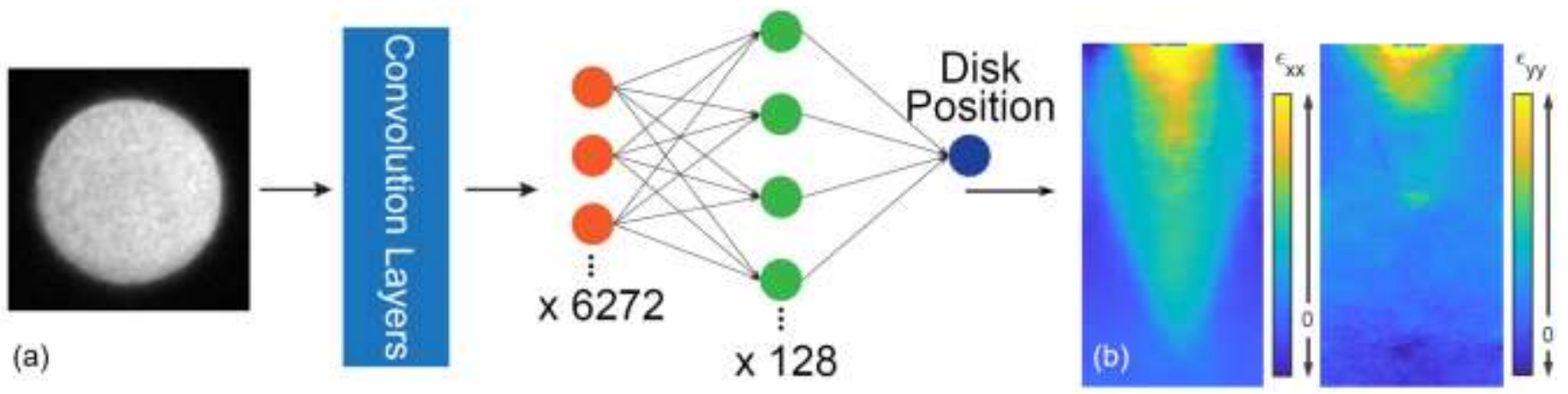

Figure 2. Training convolutional neural networks strain mapping. (a) The design of an CNN for diffraction disk position determination. The input is a $121 * 121$-pixel image containing a single diffraction disk. (b) Strain mapping of a Si-based FinFET device calculated using CNNs for diffraction disk detection.

\section{References}

[1] J.-M. Zuo, in: P.W. Hawkes, J.C.H. Spence (Eds.) Springer Handbook of Microscopy, Springer International Publishing, Cham, 2019, pp. 2-2.

[2] C. Ophus, Microscopy and Microanalysis, 25 (2019) 563-582.

[3] Tate, Mark W., et al., Microscopy and Microanalysis 22.1 (2016): 237-249; Krajnak, Matus, et al., Ultramicroscopy 165 (2016): 42-50. 
[4] Silver, David, et al., Nature 550 (2017) 354-359.

[5] Y. LeCun, et al., Nature, 521 (2015) 436-444.

[6] J.A. Aguiar, et al., Science Advances, 5 (2019) eaaw1949.

[7] C.-H. Lee, et al., Nano Letters, 20 (2020) 3369-3377.

[8] J.K. Sunde, et al., Materials Characterization, 142 (2018) 458-469.

[9] R. Yuan, et al., Ultramicroscopy, 207 (2019). 